\title{
ASSESSMENT OF THE EFFECT OF WAVE DEVICE APPLICATION ON MORPHOLOGICAL CHANGES IN ORGANS AND CELLS OF IRRADIATED ANIMALS
}

\author{
V. Bebeshko ${ }^{1, *}$, I. Homolyako', V. Grynchyshyn ${ }^{1}$ \\ ${ }^{1}$ Institute of Clinical Radiology, National Center for Scientific Research of Radiation Medicine, \\ National Academy of Medical Sciences of Ukraine, Kyiv 02000, Ukraine \\ ${ }^{2}$ O.O. Shalimov National Institute of Surgery and Transplantology, National Academy of Medical Sciences \\ of Ukraine, Kyiv 03680, Ukraine
}

\begin{abstract}
Aim: To study the effect of the Device for wave influence on biological objects on the prevention of the development of acute radiation sickness and chronic radiation syndrome in vivo. Materials and Methods: The studies were performed on white rats irradiated at a dose of $8 \mathrm{~Gy}$. The experimental group of irradiated rats was treated with a wave Device (Patent of Ukraine No. 53568) once, for $2.5 \mathrm{~min}, \mathbf{1 . 5} \mathrm{h}$ after irradiation. Their organs were processed by standard histologic methods. Results: In the demagnetized rats, dystrophic changes in cells and tissues of liver, lungs, kidneys, brain, bone marrow and spleen were insignificant in 60 days compared to the control non-demagnetized group of animals. Conclusion: The Device reduced the magnetic charge of magneto-containing elements and their compounds in the organism of the irradiated animals, and decreased the formation of reactive oxygen species, which play a key role in the development of radiation-induced diseases.
\end{abstract}

Key Words: ionizing radiation, wave Device, magnetic charge, magneto-containing compounds, radiation-induced diseases.

In the development of cell radiation injury in the dose range from 0.01 to $20 \mathrm{~Sv}$ and higher, an important role is played by oxygen, because irradiation leads to the formation of high concentrations of reactive oxygen species (ROS) - nitric oxide, peroxynitrite, primary lipid peroxidation products and toxic products of oxidative adducts of DNA. ROS arise during the process of radiation-induced hydrolysis, and begin from the formation of nonstable particles free radicals "carriers" of noncoupled electrons. They, actually, conduct this reaction "by chain" [1-4]. The latter plays the main role in the pathogenesis of radiation-induced diseases (acute radiation sickness and chronic radiation syndrome), and their oncohematological (leukemia, lymphoma, myelodysplastic syndromes, solid tumors) [6-17] and pulmonary (pneumosclerosis) complications and of more than 150 diseases, which have in the base peroxide chain processes with the formation of unstable active radicals-carriers of noncoupled electrons.

Despite the essential achievements of radiobiology and radiation medicine in diagnostics and treatment of acute and chronic radiation syndromes [5-7] and their complications, there is no effective remedy or a Device to prevent the development of these diseases. There is a lack of reliable treatment methods as well.

Device, which is protected by patent № 53568 , operates from $\sim 220 \mathrm{~V}, 50 \mathrm{~Hz}$. The device emits electromagnetic waves, which reduce the magnetic charge of magnetized elements and their compounds

Submitted: October 12, 2017.

*Correspondence: E-mail: vg.bebeshko@gmail.com Abbreviations used: MALT - mucoso-associated lymphoid tissue; ROS - reactive oxygen species. in irradiated animals and humans, resulting in decreased ROS formation in the body, which prevents the development of diseases associated with the effect of ROS.

The aim of this investigation was to assess possible effect of the use of the patent Device for intracavital influence on biological objects (Patent of Ukrainian No. 53568, V. Grynchyshyn) in in vivo model.

\section{MATERIALS AND METHODS}

The investigation was performed on white inbred rats $(m=250 \mathrm{~g}, \mathrm{n}=10)$ irradiated with a dose of $8 \mathrm{~Gy}$. The study was performed in accordance with the ethical principles for experiments on animals. The experimental group of irradiated rats $(n=5)$ were treated with a wave Device (Patent of Ukraine No. 53568) once for $2.5 \mathrm{~min}, 1.5 \mathrm{~h}$ after irradiation. Control animals $(n=5)$ received no treatment. 60 days after radiation the animals were anesthesized. Their organs (liver, kidney, brain, lung, heart, spleen, bone marrow) were processed by standard histologic methods. The paraffin slides were colored by hematoxylin and eosin. The examination and photo documentation was carried out using a BX-41 microscope (Olympus, Japan) with a micro video system and the program Quick-photo (Germany).

The changes in the organs were assessed by description and by a half-quantity method using visualanalogy scale with the step of 1 unit from 0 to $3+$. In the spleen and bone marrow we calculated the quantity of megakaryocytes as the generalized indicator of the proliferative activity in 10 visual fields at high magnification $(\times 400)$.

In the rat organs of both groups we revealed morphological changes of different intensity and dispersion; the individual variability of these changes was observed. 


\section{RESULTS AND DISCUSSION}

The rats liver showed considerable proteinhydropic dystrophy, which in the control group attained point $3+$ with separate small areas of hepatocyte lysis and intralobular necrosis. Dystrophic changes and considerable nuclear polymorphism with the prevalence of small nuclei, the phenomenon of pyknosis and apoptosis testified the pronounced intoxication and hypoxia. Inflammatory infiltration was insignificant and might be observed only in separate portal tracts, it was accompanied by a pronounced reaction of the liver macrophages.

Sometimes slight manifestations of pyle phlebitis were seen. The results of a half-quantitative analysis in general showed, that the degree of liver damage in the control group exceeded significantly the main group (54 vs 31 points, respectively). So, the intensity of degenerative processes in the liver under the influence of the Device decreased by 1.43 times (Fig. 1, 2).

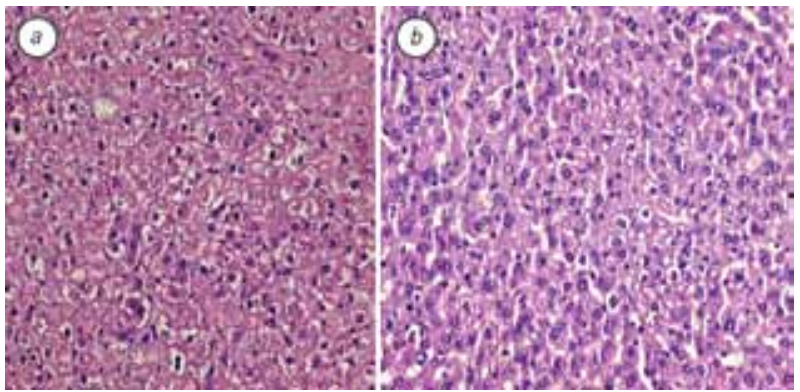

Fig. 1. The liver slide of the radiation-exposed animal: significant protein-hydropic dystrophia with hepatocyte nuclear polymorphism (a); the liver slide of irradiated animal treated with the Device structure of liver is close to the normal one $(b)$. Hematoxylin-eosin, $\times 400$

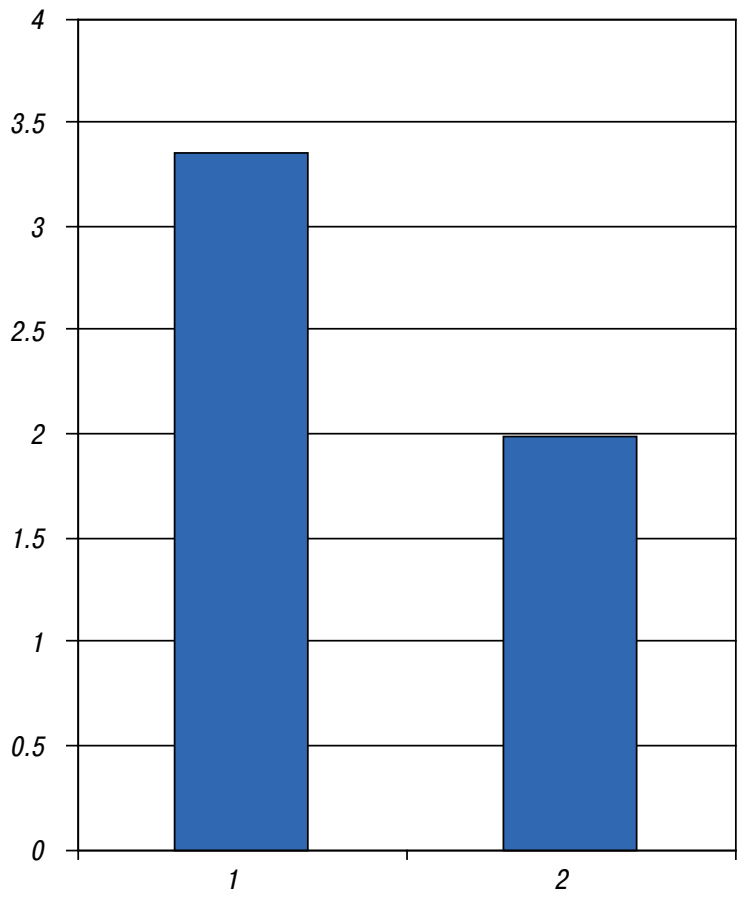

Fig. 2. The significance of dystrophia in the liver of the irradiated animals (1) is much higher than in the liver of the irradiated animals treated with the Device (2)

Visible enough was also the reaction of the spleen. The ratio of white and red pulp in the control rats was
0.78 according to point scoring. The volume of red pulp was higher than the volume of white pulp. The quantity of megakaryocytes calculated in the 10 visual fields in high magnification was 0.38 . This is evidence of low proliferative activity of lymphoid tissue. Moreover, the devastation, atrophy, edema of red pulp and sinuses were observed.

The Device contribute to a slight change of the ratio between white and red pulp volumes (0.76). The volume of red pulp was higher than the volume of white pulp, but the quantity of lymphoid cells increased, the index of the quantity of megakaryocytes which amounted to 0.84 also increased considerably, which testified the intensification of proliferative activity of cells in the lymphoid and haemopoetic tissue (Fig. 3, 4).

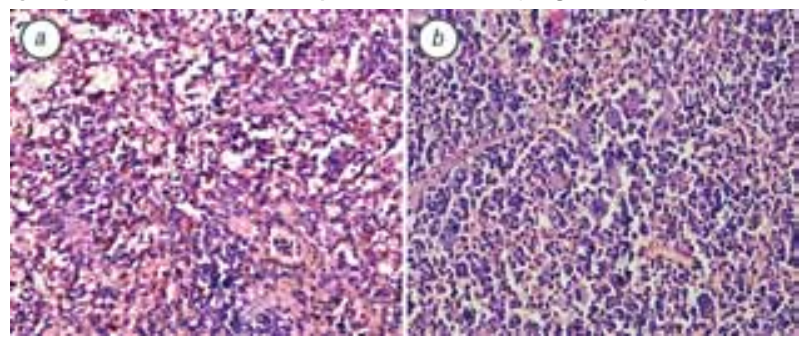

Fig. 3. The slide of the spleen of the irradiated animal: swelling and atrophic changes in the red pulp, plethora of sinuses of the red pulp (a); the slide of the spleen of the radiation-exposed animal treated with the Device: the increase of the cellularity, group of functionally retained megakaryocytes in the spleen $(b)$. Hematoxylin-eosin, $\times 400$

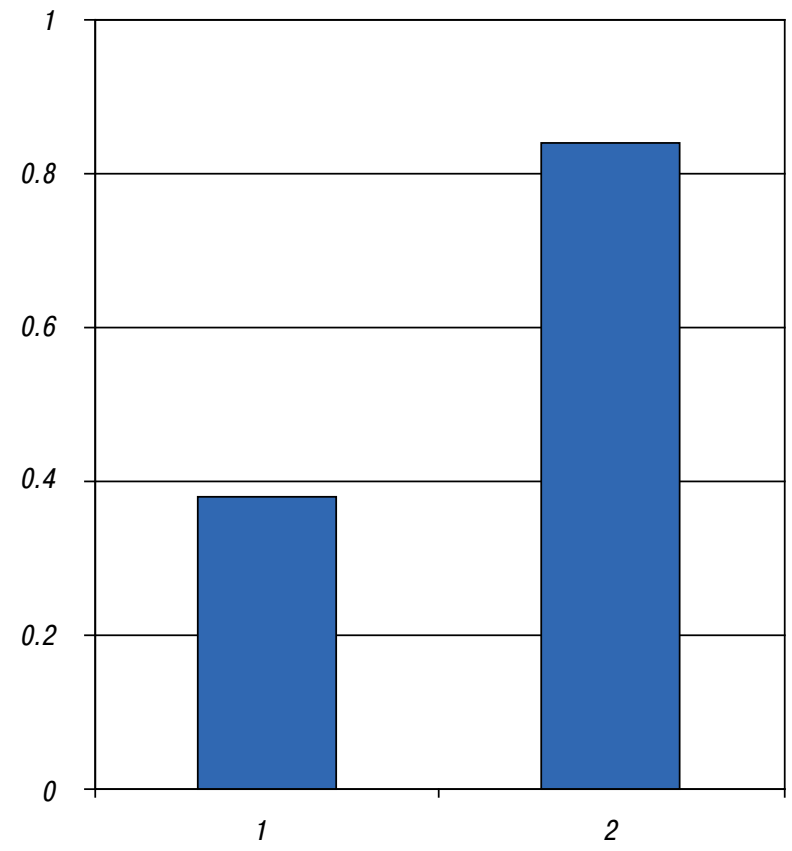

Fig. 4. The increase of the quantity of retained megakaryocytes in the spleen of the irradiated animals (1) and in spleen of the radiation-exposed rats with the use of the Device (2)

The comparison of the state of the bone marrow was also carried out by the description of megakaryocyte quantity. In rats of the main group under the influence of the Device, we saw a decreased quantity of dilated plethoric capillaries, increased cellularity and safety of bone marrow cells; the middle quantity of megakaryocytes stayed fixed -14.6 (control), 17.2 (the main group) (Fig. 5). 


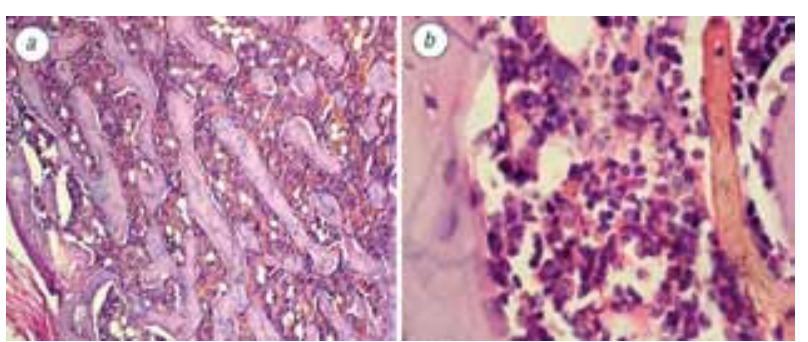

Fig. 5. The bone marrow of the radiation-exposed animals intensive vascularisation (a); the bone marrow of the radiationexposed animals after the use of the Device the increase of cellularity, retained megakaryocytes $(b)$. Hematoxylin-eosin, $\times 100(a), \times 400(b)$

The distinguished features of the reaction of the pulmonary tissue on the radiation and on the radiation with the use of the Device were intrinsic and obvious.

After irradiation in the rats we have observed the focal pneumonia, focal seats of stress reaction dilated plethoric capillaries of the intra-alveolar septa, extravasates, the seats of edema, the diffuse and seat thickening of the intra-alveolar septa with histiocytic and macrophagal infiltration. The intensity of these changes varied from moderate to pronounced. Mucoso-associated lymphoid tissue (MALT) had signs of atrophy and a decrease of activity as a result of the decreased volume of lymphoid tissue and absence of germinal centers (Fig. 6).

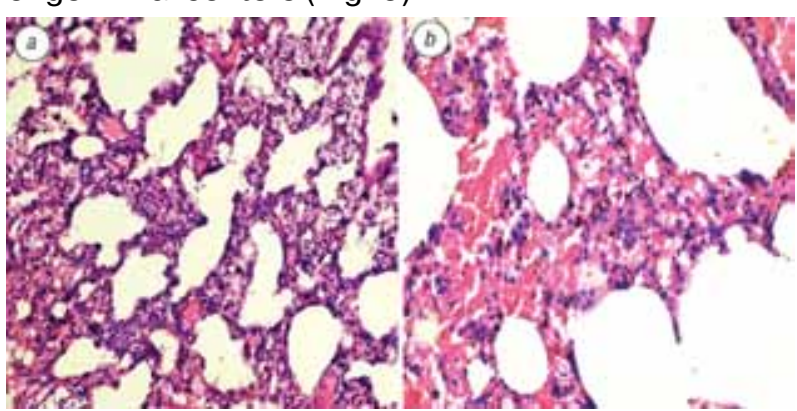

Fig. 6. The slides of the lungs of radiation-exposed animals: alveolar septae are thickened, infiltrated with histiomacrophagal elements, capillaries are dilated (a); focus of clear dilatation of capillaries with haemorrhage $(b)$. Hematoxylin-eosin, $\times 200(a), \times 400(b)$

The employment of the Device lead to a decrease of lung damage. The focuses of pneumonia had a small size and of insignificant intensity, and were revealed only in some animals. In the majority of the observations the lung had an ordinary structure with single areas of atelectases, dystelectases and emphysema. MALT also returned to normal. In some animals the perivascular lymphoid infiltration, which is evidence of the delayed hypersensitivity, was seen (Fig. 7, Fig. 8).The kidneys of the rats from both groups showed similar changes - insignificant dilatation and congestion of the vessels, some hemorrhages. The injure of glomeruli was represented by hyper cellularity and the collapse of the capillaries. Tubules were normal or dilated, with the focuses of protein-hydropic or sometimes fatty dystrophy. These changes were more typical for the control group.

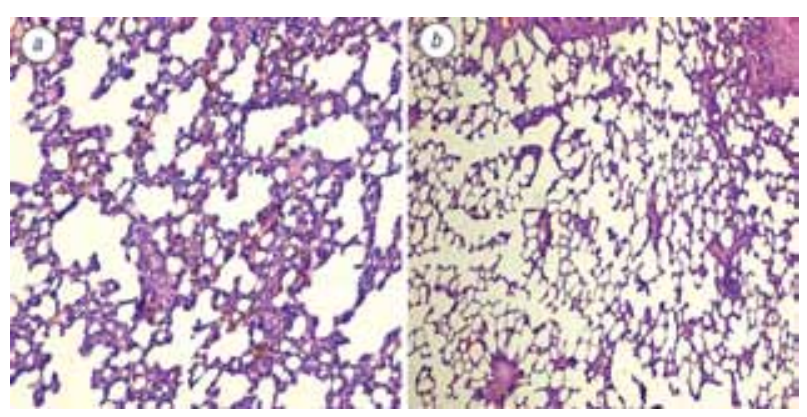

Fig. 7. The slides of the lungs of the radiation-exposed animals treated with the Device: focuses of the slight thickening of alveolar septae, infiltration with histiomacrophagal elements, capillaries are dilatation (a); structure of the lung is close to the normal one $(b)$. Hematoxylin-eosin, $\times 100(a)$ and $\times 40(b)$

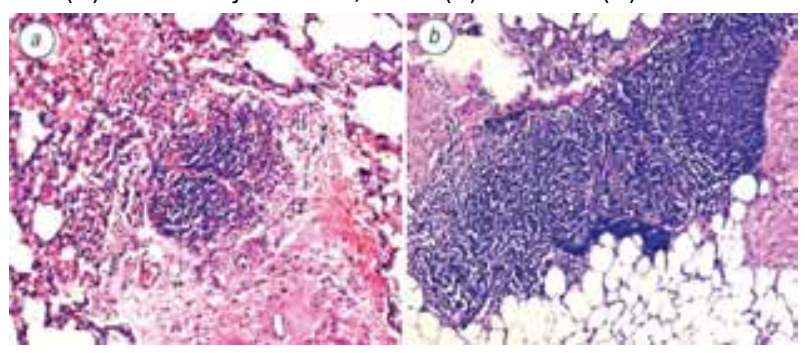

Fig. 8. MALT of the radiation-exposed animals with the signs of reduction (a); MALT of the radiation-exposed animals treated with the Device; significant hyperplasia, reactive changes, large reactive centers $(b)$. Hematoxylin-eosin, $\times 200$

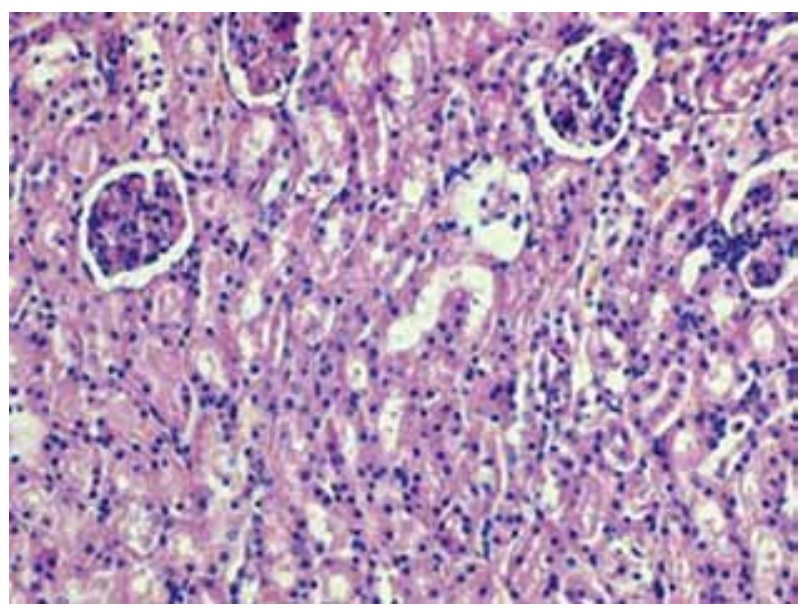

Fig. 9. The slide of the kidney of the radiation-exposed animal: protein-hydropic dystrophy of the tubular epithelium, increase of glomerulus cellularity, the collapse of glomerular capillaries. Hematoxylin-eosin, $\times 100$

The general alignment of glomeruli injure in control group was 3.36, in the main group 1.99 (Fig. 9, 10).

The structure of heart muscle and the structure of the brain in both groups were in normal range.

It's necessary to pay attention to the following. In the rats irradiated by the 8 Gy dose (control group) after the start of the experiment in all the organs were observed noticeable pathologic changes, "vascular reaction" (dilatation of vessels, plethoric, stasis, hemorrhage) and noticeable dystrophic changes. After the use of the Device the intensity of the vascular reactions and dystrophy decreased. In the liver the results of a half-quantity appreciation of intensity of protein-hydropic dystrophy and other manifestations of dystrophy decreased by 1.43 times. In the 


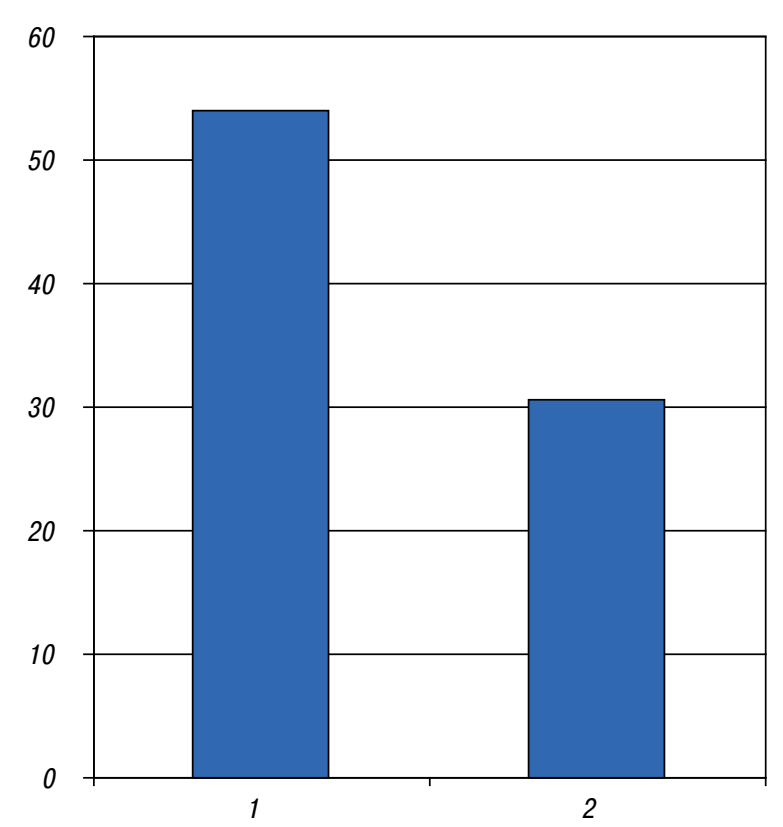

Fig. 10. The rate of damaged glomeruli in the kidney of the radiation-exposed animals (1) and of the radiation-exposed animals treated with the Device (2)

spleen the alignment of the white and red pulp in the control and the main group had no significant difference ( 0.78 and 0.76 , accordingly). But the index of the quantity of megakaryocytes increased from 0.38 to 0.84 . It reflects the essential intensification of the proliferative reaction in the lymphoid and hemopoietic organs. Also, we could see the normalization of structure of the red pulp.

The significant changes in the bone marrow were observed. In the control group, mainly "fatty" bone marrow was observed with middle intensity pathologic changes in the elements of granulocytes and thrombocytes lineage. In the bone marrow of the main group, the restoration of the cells of these lineages and the cells of erythroid lineage was higher in the "niche" (3+), the intercellular connections were not destroyed and the morphologic characteristics corresponded to the normal state in intact rats. The quantity of megakaryocytes in the marrow in the main group was sometimes higher (17.2 vs 14.6 in control).

In the kidneys of the rats of the main (experimental) group the parenchyma damage was also not considerable. The middle ratio between damaged and intact glomeruli in the control group was 3.36, in the main group - 1.99. It means that in the main group the quantity of the damaged glomeruli was significantly lower. Moreover, the signs of the protein-hydropic and fatty dystrophy in the tubular epithelium and vascular reactions were absent. The distinguishing features of the lung reactions to the irradiation and on the irradiation with the using of the Device are significant. In the animals after the irradiation we could see the centers of pneumonia, the manifestations of stress reaction hemorrhage, delatation of capillaries of the interalveolar barrier, extravasates, edema, diffuse and the local thickening of the inter-alveolary barrier with the infiltration of hystiocytes and makrophages.
The use of the Device led to a significant decrease of lung damage and in most observations the lung showed the normal structure with the single focuses of atelectases, dystelectases, and emphysema. These changes might be a concern to the ordinary function of the lung or to the methods of avtanasia.

In irradiated animals MALT had the significant signs of the reduction and decrease of activity: the decrease of the volume of lymphoid tissue, absence of germinative centers. In the main group, the normal structure of MALT was seen. Some animals demonstrated perivascular lymphoid infiltration - the manifestation of the delayed hypersensitivity. The structure of the heart muscle and structure of the brain in the both groups were in the limits of normal deviations.

In summary, experimental study demonstrated that Device for wave influence on biological objects decreased the development of pathological processes and, consequently, affected morphological changes in the most radiosensitive organs (bone marrow, spleen, lungs, etc.) in experimental animals.

The results of our investigations allow to recommend the Device for use by the workers in the nuclear industry, participants of the liquidation of the consequences of a techno-radiation accidents, radiologists, radiobiologists and other workers, who work with magnetic irradiators to prevent the development of radiation-induced diseases and their complications. We consider that this wave device might to use it in the treatment of oncohaematological diseases that are treated using radiation and cytostatic drugs, which increase the concentration of ROS in the body of patients, the sources of disease recurrence and the development of secondary tumors.

\section{REFERENCES}

1. Kudryashov YuB. Radiation biophysics (Ionizing Radiation). New York: Nova Science Publishers Inc, 2008. 327 p.

2. Valentin J. Low-dose extrapolation of radiation-related cancer risk. Ann ICRP 2005; 35: 1-140.

3. Classification of chronic gastritis. Doctor, 2000; (3): 18-19.

4. Tashke K. Introduction to Quantitative Cytological Histology Morphology, 1980. 191 p.

5. Bebeshko V, Belyi D, Kovalenko A, et al. Health consequences in the Chernobyl emergency works surviving after confimed acute radiation sickness, pp. 5-26. B: Followup of Delayed Health Consequences of Acute Accidental Radiation Exposure. Lessons to be Learned From their Medical Management. IAEA-TECDOC-1300. IAEA, Vienna (2002).

6. Preston D, Kusumi S, Tomonaga M, et al. Cancer incidence in atomic bomb survivors. Part III. Leukemia, lymphoma and multiple myeloma, 1950-1987. Radiat Res 1994; 137 (2 Suppl): 68-97.

7. Mabuchi K, Kusumi S. Leukemia. In: Effects of A-Bomb Radiation on the Human Body. Shigematsu I, Ito C, Kamada N, et al., eds. Singapore: Harwood Acad Publ GmbH, 1995: 40-4.

8. Fliedner TM, Densow D, Fischer B, et al. Evaluation of acute radiation syndrome patients with computerized database. Joint study project No 3. Diagnosis and treatment of patients with acute radiation syndrome. Final report. Wagemaker G, Bebeshko VG, eds. Luxembourg: European Commission, 1996: 25-37. 
9. Romanenko A, Bebeshko V, Hatch M, et al. The ukrainian-american study of leukemia and related disorders among Chornobyl cleanup workers from Ukraine: I. Study methods. Radiat Res 2008; 170: 691-7.

10. Pukkala E, Kesminiene A, Poliakov S, et al. Breast cancer in Belarus and Ukraine after the Chernobyl accident. Int J Cancer 2006; 119: 651-8.

11. Tokunaga $M$, Land CE, Tokuoka $S$, et al. Incidence of female breast cancer among atomic bomb survivors, 1950-1985. Radiat Res 1994; 138: 209-23.

12. Bebeshko VG, Bruslova EM, Klimenko VI, et al. Leukemia and lymphomas at population of Ukraine exposed to chronic low dose irradiation. In: Low doses of ionizing radiation: biological effects and regulatory control. Vienna: IAEA, 1997: 871-8.
13. Zablotska LB, Bazyka D, Lubin JH, et al. Radiation and the risk of chronic lymphocytic and other leukemias among Chornobyl cleanup workers. Environ Health Perspect 2013; 121: 59-65.

14. Doll RS. Hazards of ionising radiation: 100 years of observations on man. Br J Cancer 1995; 72: 1339-49.

15. Yoshinaga S, Mabuchi K, Sigurdson AJ, et al. Cancer risks among radiologists and radiologic technologists: review of epidemiologic studies. Radiology 2004; 233: 313-21.

16. Effects of A-bomb radiation on the human body. Shigematsu I, Ito C, Kamada N, et al., eds. Singapore: Harwood Acad Publ GmbH, 1995. 419 p.

17. Gluzman DF. Leukemias and myelodysplastic syndromes following the Chernobyl accident. Exp Oncol 1996; 18: $120-7$. 